\title{
Call for experimental test of a revised mathematical form for empirical field emission current-voltage characteristics
}

\author{
Richard G. Forbes ${ }^{\text {a) }}$ \\ Advanced Technology Institute (X1), Faculty of Engineering and Physical Sciences, University of Surrey, \\ Guildford, Surrey GU2 7XH, United Kingdom
}

(Received 1 January 2008; accepted 10 April 2008; published online 14 May 2008)

\begin{abstract}
There are now good theoretical reasons to believe that experimental current-voltage $(i-V)$ relationships for cold field electron emission (CFE) should approximately obey the empirical law $i=C V^{\kappa} \exp (-B / V)$, where $B, C$, and $\kappa$ are effectively constant, and $\kappa \neq 2$. Since $-d \ln i / d(1 / V)$ $=\left(V^{2} / i\right) d i / d V=B+\kappa V, \kappa$ for a given emission situation could be experimentally established from an appropriate data plot if sufficiently accurate current-voltage measurements were available. Knowledge of experimental $\kappa$ values could lead to greater physical understanding of CFE and variations as between materials. It is strongly urged that the feasibility of measurements of this kind be carefully explored. (C) 2008 American Institute of Physics. [DOI: 10.1063/1.2918446]
\end{abstract}

Fowler-Nordheim (FN) tunneling ${ }^{1}$ is electric-fieldinduced electron tunneling through a roughly triangular potential-energy barrier. When the barrier is strong (so the tunneling probability is small, say $10 \%$ or less), a lowtemperature emission regime (including room temperature) exists, called "cold field electron emission" (CFE).

A critical step in understanding CFE was the discovery of Millikan and Lauritsen ${ }^{2}$ that experimental current-voltage $(i-V)$ characteristics plotted as $\left[\log _{10}(i)\right.$ versus $\left.1 / V\right]$ are good straight lines. This suggested the empirical equation

$$
i=C \exp (-B / V) \text {, }
$$

where $B$ and $C$ are effectively constant.

FN's well-known theory ${ }^{1}$ uses the physical simplification that tunneling takes place from a flat planar surface, through an exact triangular (ET) barrier. This led (after correction ${ }^{3}$ ) to the "original FN-type equation," written here

$$
J=a \phi^{-1} F^{2} P_{F} \exp \left(-b \phi^{3 / 2} / F\right) .
$$

This gives the emission current density $J$ in terms of the local emitter work-function $\phi$ and surface field $F$. The symbols $a\left(\cong 1.541434 \times 10^{-6} \mathrm{~A} \mathrm{eV} \mathrm{V}^{-2}\right)$ and $b\left(\cong 6.830890 \mathrm{eV}^{-3 / 2} \mathrm{~V} \mathrm{~nm}^{-1}\right)$ denote universal constants, called the first and second FN constants. ${ }^{4} P_{F}$ is the tunneling prefactor

$$
P_{F}=4 \phi^{1 / 2} K_{F}^{1 / 2} /\left(\phi+K_{F}\right),
$$

where $K_{F}$ is the Fermi energy. $P_{F}$ is usually of order unity and CFE literature often ignores it. Setting $P_{F}=1$ in Eq. (2) gives the "elementary FN-type equation."

With the further assumptions that $F=\beta V$, where $\beta$ is a constant, and that emission area $A$ is constant in the relation $i=A J$, Eq. (2) suggests that empirical CFE $i-V$ characteristics should obey

$$
i=C V^{\kappa} \exp (-B / V),
$$

where $B, C$, and $\kappa$ are constants and $\kappa=2$. The discrepancy between forms (1) and (4) was thought unimportant by FN (Ref. 1) because 1920s CFE experiments could not distinguish between them. Due to Eq. (2), it became customary to

\footnotetext{
${ }^{a)}$ Electronic mail: r.forbes@ieee.org.
}

plot CFE results as a "FN plot" $\left[\ln \left(i / V^{2}\right)\right.$ versus $\left.1 / V\right]$, on the grounds that this should generate an exact straight line.

In 1939, Abbott and Henderson ${ }^{5}$ argued that the emission area $A$, and the correction factor $\nu_{F}^{\mathrm{SN}}$ discussed below, might be functions of field and voltage. So, an experimental value $\kappa=2$ was not necessarily expected. They made empirical data plots of form $\left[\log _{10}\left(i / F^{\kappa}\right)\right.$ versus $\left.1 / F\right]$ for the values $\kappa=0,2,3,4$. Deviation of the plot from a straight line was least for $\kappa=4$. This result has no obvious theoretical explanation and has largely been ignored.

Taking the tunneling barrier as exactly triangular is simple and mathematically convenient but not physically realistic. The next simplest approximation includes a classical image potential energy. For a barrier of zero-field height $\phi$, the variation in electron energy $M$ with position is then

$$
M(z)=\phi-e F z-e^{2} / 16 \pi \varepsilon_{0} z,
$$

where $z$ is distance from the emitter's electrical surface ${ }^{6}$ and $\varepsilon_{0}$ is the electric constant. This barrier, introduced by Schottky ${ }^{7}$ and used by Nordheim, ${ }^{8}$ has been called a "Schottky-Nordheim" (SN) barrier. Its use in CFE theory causes $^{4}$ the appearance, in the exponent of a theoretical FNtype equation, of a physical "barrier-shape correction factor," denoted here by $\nu_{F}^{\mathrm{SN}}$. Thus, for the SN barrier, physical theory predicts an exponent of form: $\exp \left[-\nu_{F}^{\mathrm{SN}} b \phi^{3 / 2} / F\right]$. Typically, $\nu_{F}^{\mathrm{SN}}$ has value around 0.7 and makes the predicted current density higher by a factor of around 100 .

Recent mathematical developments in CFE theory, ${ }^{4,9}$ have yielded a good, simple approximate formula for the correction factor $\nu_{F}^{\mathrm{SN}}$, namely,

$$
\nu_{F}^{\mathrm{SN}} \approx 1-F / F_{\phi}+(1 / 6)\left(F / F_{\phi}\right) \ln \left(F / F_{\phi}\right),
$$

where $F_{\phi}$ is the physical field necessary to reduce to zero a barrier originally of height $\phi$. We cannot directly measure $F_{\phi}$ but can estimate it using Schottky's formula: ${ }^{10}$ $F_{\phi}=\left(4 \pi \varepsilon_{0} / e^{3}\right) \phi^{2}$. Equation (6) outperforms all existing approximations of equivalent complexity. ${ }^{4}$

Inserting Eq. (6) into the exponent of Eq. (2) and also making the conventional (but usually hidden) approximation $P_{F}=1$ yields 


$$
J=\left(a \phi^{-1} \mathrm{e}^{\eta} F_{\phi}^{\eta / 6}\right) F^{(2-\eta / 6)} \exp \left(-b \phi^{3 / 2} / F\right),
$$

where $\eta\left(\equiv b \phi^{3 / 2} / F_{\phi}\right)$ is a constant. Since $F \propto V$, Eq. (7) clearly predicts that, in the empirical equation (4), $\kappa \neq 2$. For $\phi=4.5 \mathrm{eV}$, we have $F_{\phi}=14.0 \mathrm{~V} / \mathrm{nm}, \eta=4.64$, and $\kappa=2-\eta / 6=1.2$.

More generally, there are other theoretical reasons to expect that $\kappa \neq 2$. (a) It is obvious from field electron microscope observations that sometimes the emission area $A$ is a function of applied voltage. (b) Jensen ${ }^{11}$ showed that $\phi$ should be a weak function of $F$. (c) The term $F^{2}$ in the pre-exponential of Eq. (2) is obtained by integrating contributions from all electronic states associated with a bulk, three-dimensional, free-electron metal. Other materials, in particular carbon nanotubes, may have significantly different distributions of electronic states; so the integration might yield a different power of $F$ (and, hence, $V$ ) in the preexponential.

The above suggests fitting experimental data with Eq. (4), taking $\kappa$ as unknown. One could try plotting $\ln \left(i / V^{\kappa}\right)$ versus $1 / V$. However, this is not a good method when $\kappa$ is unknown and probably nonintegral. In principle, an alternative exists. From Eq. (4),

$$
\begin{aligned}
& \ln (i)=\ln (C)-\kappa \ln (1 / V)-B / V, \\
& -d \ln (i) / d(1 / V)=\kappa V+B .
\end{aligned}
$$

So, in principle, $\kappa$ can be obtained from the slope of the plot: $-d \ln (i) / d(1 / V)$ versus $V$.

If we write $F=\beta V$, where $\beta$ is constant, and also assume emission area $A$ is constant, then the $J-F$ equation equivalent to Eq. (9) is

$$
-d \ln (J) / d(1 / F)=\kappa F+\beta B .
$$

The plotting method based on Eq. (10) was tested on simulated data sets, for an ET barrier and for a SN barrier. First, data sets of $\ln (J)$ versus $1 / F$ were prepared via the commonly used equations based on these barriers, namely, the elementary FN-type equation obtained from Eq. (2) by setting $P_{F}=1$, and the so-called standard FN-type equation developed by Murphy and Good, ${ }^{12}$ evaluated as described in Ref. 13. The value $\phi=4.5 \mathrm{eV}$ was used. The values of $\ln (J)$ were obtained at equally spaced values of $F$, from 3.4 to $10.2 \mathrm{~V} / \mathrm{nm}$. The $m$ th value of $-d \ln (J) / d(1 / F)$ was obtained as a local average, from the $(m-1)$ th and $(m+1)$ th values of $1 / F$ and $\ln (J)$.

Figure 1 shows the results of plotting $-d \ln (J) / d(1 / F)$ versus $F$. For both the ET and SN barriers, the plots are good straight lines. The extracted slope $(\kappa)$ values are 2.0 for the ET barrier, as expected, and 1.1 for the SN barrier; this adequately agrees with the value of 1.2 that was predicted from Eq. (7). These agreements clearly show that this method works in principle.

To test it on experimental results, the data in Fig. 3 of Ref. 14 were digitized and processed. (These data are a well characterized set used in 1953 to test the validity of CFE theory. ${ }^{14}$ ) Figure 2 shows that the resulting plot is too noisy for useful conclusions. There seem problems in both the original data and the digitization. Clearly, this method needs raw current-voltage data from careful experiments in lownoise conditions. I cannot find suitable tabulations in $\mathrm{CFE}$

literature.
Downloaded 30 Mar 2009 to 131.227.178.132. Redistribution subject to AlP license or copyright; see http://apl.aip.org/apl/copyright.jsp

\section{$-d \ln \{\boldsymbol{J}\} / \mathrm{d}(\mathbf{1} / \boldsymbol{F}) \quad\left[\boldsymbol{J}\right.$ in $\mathrm{A} / \mathrm{m}^{2} ; \boldsymbol{F}$ in $\left.\mathrm{V} / \mathrm{nm}\right]$}

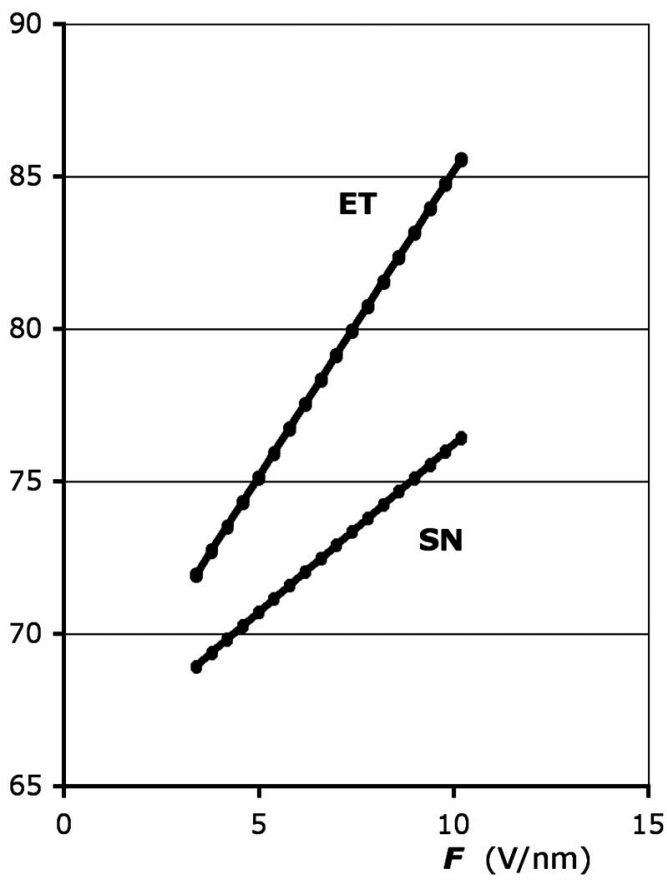

FIG. 1. Equation (10) plotted for simulated data corresponding to an ET barrier and a SN barrier.

With modern computer-controlled equipment, voltage setting and current measurement will be achievable with good accuracy, provided that the electronics has high stability and low noise, and the emission itself is stable (which requires good ultrahigh vacuum). Errors in finding small differences in $\ln (i)$ could be a problem. So, the alternative form of Eq. (9) may be better,

$$
\left(V^{2} / i\right) d i / d V=B+\kappa V,
$$

with $d i / d V$ found by phase-sensitive detection techniques, as used (for example) by Spindt et al. ${ }^{15}$

If accurate measurements are proven possible, then, to interpret results, one needs to establish which physical factors are most likely to influence $\kappa$. For a bulk metal emitter, the voltage-to-local-field conversion factor $\beta$ can normally be treated as constant (but this may not be true for nonmetallic emitters). At present, the exponent correction factor and the emission area seem the most influential.

Two investigations would clearly be of interest. (1) Experiments where the apparent emission area can be measured accurately and kept constant as voltage changes. One ap-

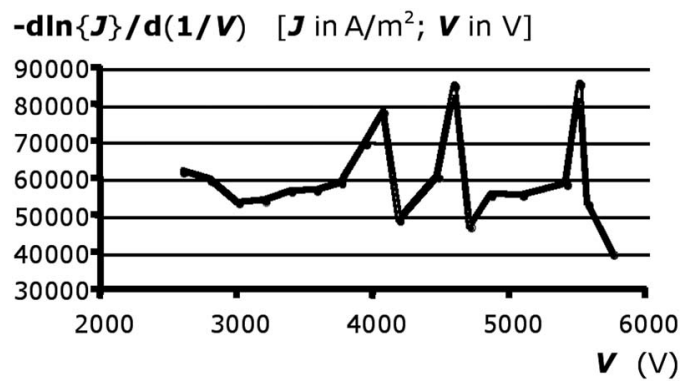

FIG. 2. Equation (9) plotted for experimental data relating to a tungsten emitter of radius $220 \mathrm{~nm}$, digitized from Fig. 3 of Ref. 14. Two obviously

deviant points were omitted but noise makes the plot of limited use.
o AIP license or copyright; see http://apl.aip.org/apl/copyright.jsp 
proach is to use a field ion microscope, with a probe hole aligned over a well-defined part of the image (e.g., over a known number of atomic sites in a net plane). Here, one might learn if the SN barrier is a good model for the actual surface barrier. (2) From experiments on the total emission from a tip of known shape, one might learn about the dependence of emission area on applied voltage.

Also of interest are experiments on materials where the emitted electrons are not from a bulk metal conduction band. This is because (as noted above) the integration over electronic states may give a different power-law dependence in the pre-exponential. Of particular interest would be experimental $\kappa$ values for emission from: (a) semiconductor surface states; ${ }^{16}$ (b) a Fink-type single-atom electron emitter, ${ }^{17}$ and/or a built-up pyramidal three-atom emitter as used in helium scanning ion microscopy; ${ }^{18}$ and (c) open and closed single-walled carbon nanotubes of known configuration and radius.

Determination of $\kappa$ values could be an extra experimental route to greater understanding of CFE and its variation as between different materials. I strongly urge that it would be useful to explore whether the proposed measurements can be effectively carried out. If so, it would also be useful to have the resulting raw $i-V$ data available either in print or as supplementary electronic data.

${ }^{1}$ R. H. Fowler and L. W. Nordheim, Proc. R. Soc. London, Ser. A 119, 173 (1928).

${ }^{2}$ R. A. Millikan and C. C. Lauritsen, Proc. Natl. Acad. Sci. U.S.A. 14, 45 (1928).

${ }^{3}$ T. E. Stern, B. S. Gossling, and R. H. Fowler, Proc. R. Soc. London, Ser. A 124, 699 (1929).

${ }^{4}$ R. G. Forbes and J. H. B. Deane, Proc. R. Soc. London, Ser. A 463, 2907 (2007).

${ }^{5}$ F. R. Abbott and J. E. Henderson, Phys. Rev. 56, 113 (1939).

${ }^{6}$ N. D. Lang and W. Kohn, Phys. Rev. B 7, 3541 (1973).

${ }^{7}$ W. Schottky, Phys. Z. 15, 872 (1914).

${ }^{8}$ L. W. Nordheim, Proc. R. Soc. London, Ser. A 121, 626 (1928).

${ }^{9}$ R. G. Forbes, Appl. Phys. Lett. 89, 113122 (2006).

${ }^{10}$ W. Schottky, Phys. Z. 14, 63 (1923).

${ }^{11}$ K. L. Jensen, J. Appl. Phys. 85, 2667 (1999).

${ }^{12}$ E. L. Murphy and R. H. Good, Phys. Rev. 102, 1464 (1956).

${ }^{13}$ R. G. Forbes, J. Vac. Sci. Technol. B 17, 526 (1999).

${ }^{14}$ W. P. Dyke and J. K. Trolan, Phys. Rev. 89, 799 (1953).

${ }^{15}$ C. A. Spindt, I. Brodie, L. Humprey, and E. R. Westerberg, J. Appl. Phys. 47, 5248 (1976)

${ }^{16}$ A. Modinos, Surf. Sci. 42, 205 (1974).

${ }^{17}$ H.-W. Fink, Phys. Scr. 38, 260 (1988).

${ }^{18}$ B. W. Ward, J. A. Notte, and N. P. Economu, J. Vac. Sci. Technol. B 24, 2871 (2006). 